Ebisu Ebisu

Études japonaises Études japonaises

$58 \mid 2021$

2011-2021 : crises, ruptures et nouvelles dynamiques.

Dix ans après la triple catastrophe du 11 mars

\title{
Crise alimentaire, sécurité sanitaire et qualité des aliments : l'anormal retour à la normale?
}

\section{Nicolas Baumert}

\section{OpenEdition}

\section{Journals}

Édition électronique

URL : https://journals.openedition.org/ebisu/5937

DOI : $10.4000 /$ ebisu. 5937

ISSN : 2189-1893

Éditeur

Institut français de recherche sur le Japon à la Maison franco-japonaise (UMIFRE 19 MEAE-CNRS)

Édition imprimée

Date de publication : 1 novembre 2021

Pagination : 167-185

ISSN : $1340-3656$

Référence électronique

Nicolas Baumert, «Crise alimentaire, sécurité sanitaire et qualité des aliments : I'anormal retour à la normale? », Ebisu [En ligne], 58 | 2021, mis en ligne le 01 novembre 2021, consulté le 13 avril 2022. URL : http://journals.openedition.org/ebisu/5937 ; DOI : https://doi.org/10.4000/ebisu.5937 


\section{Crise alimentaire, sécurité sanitaire et qualité des aliments : l'anormal retour à la normale?}

Nicolas BAUMERT*

Dès l'annonce de l'accident de la centrale de Fukushima en mars 2011, il y eu une forte inquiétude quant à la contamination de l'alimentation et cette question fut paradoxalement bien plus médiatisée que les destructions matérielles et les pertes humaines du séisme et du tsunami. Après les craintes suscitées par le passage des nuages radioactifs, le rapport à la nourriture a été la seconde source de préoccupation, mettant en avant un décalage entre, d'une part, les mesures prises par les autorités japonaises et leur discours rassurant et, d'autre part, la défiance d'une partie des consommateurs.

Les craintes ont été d'autant plus fortes que l'angoisse et l'incertitude des humains face à la nourriture sont des faits anthropologiques (Fischler 2001) qui s'observent, malgré les avancées de la connaissance scientifique, tout au long de l'histoire humaine (Ferrière 2002). La contamination radioactive est peut-être de ce point de vue l'une des plus anxiogènes, puisqu'elle combine l'image de la puissance de destruction du nucléaire et la conscience de la longue durée d'une menace pouvant potentiellement provoquer des maladies graves et des morts atroces (leucémies, cancers). Cette menace est singulière, d'abord parce que d'origine humaine, non naturelle, mais aussi parce que rien dans l'apparence ou le goût des aliments ne permet de l'identifier. La combinaison de ces éléments donne ainsi un potentiel anxiogène très élevé, ce qui explique que les consommateurs japonais se soient montrés méfiants et que la plupart des États aient rapidement interdit l'importation

* Maître de conférences à l'université de Nagoya. 
de produits agricoles japonais et demandé des tests, y compris ceux pour qui ces importations étaient anecdotiques.

La réaction de peur, ou du moins de méfiance, se fonde sur l'incertitude des conclusions de la science, sur la parole pas toujours facile à comprendre des experts, mais aussi sur une vision déformée de la réalité de l'agriculture moderne. Pour simplifier, l'image qui a véhiculé la peur est que l'agriculture japonaise nourrissait toujours l'Archipel et que par causalité directe, si les sols et la mer étaient contaminés, l'ensemble de l'alimentation, issue des cultures et de la pêche, serait dangereuse pour la consommation humaine. En réalité, le taux d'autosuffisance alimentaire en calories annuelles du Japon étant inférieur à $40 \%$ (chiffre qui évolue peu depuis une vingtaine d'années) ${ }^{1}$, même si une grande partie de l'agriculture japonaise avait été touchée avec des régions entières incultivables, les conséquences auraient finalement été limitées pour l'alimentation quotidienne à l'échelle du pays. Hormis le riz, au Japon, presque tout est massivement importé, y compris l'alimentation du bétail.

Le décalage entre la réalité du risque, les discours officiels et la perte de confiance des consommateurs dans la sécurité sanitaire des aliments constitue le fondement d'une crise alimentaire. Alors que les questions de sécurité alimentaire évoquaient jusque dans les années 1990 principalement les risques de famine, de sous-alimentation ou de malnutrition, le contexte des sociétés développées où les dangers ne sont plus liés au manque de nourriture mais à leur qualité sanitaire a conduit à une nouvelle acception des crises, prenant en compte la qualité sanitaire des aliments et sa perception par les consommateurs (Apfelbaum 1998; Poulain 2012). Si, dix ans après l'accident, il semble ne pas y avoir eu de conséquences directes sur la santé des populations en lien avec leur alimentation (UNSCEAR 2020), il y a tout de même eu, à la suite de la contaminarion radioactive, de vraies incertitudes par rapport à l'alimentation quotidienne, et ce bien au-delà des zones proches de la centrale accidentée. Des cas avérés de produits contaminés qui se sont retrouvés dans la chaine alimentaire ont aussi été rapportés, ce qui a entraîné des réactions très contrastées de la part des

1. Données du ministère de l'Agriculture, des Forêts et de la Pêche (Nōrin suisanshō 農林水産省), disponibles sur le site https://www.maff.go.jp/j/tokei/sihyo/data/02.html (dernière consultation en juin 2021). 
consommateurs. Ces différents éléments permettont, après avoir analysé l'évolution du décalage des perceptions selon le modèle de la crise alimentaire, de se poser des questions plus générales sur le rapport des Japonais à leur terre via leur agriculture et la médiation alimentaire.

\section{La crise alimentaire issue de la contamination radioactive de mars 2011 : une situation non anticipée}

L'accident de la centrale de Fukushima en mars 2011 a entraîné une contamination à des degrés variables de $10 \%$ du territoire japonais, mais une situation bien plus grave a néanmoins été évitée, puisque $70 \%$ à $80 \%$ des rejets atmosphériques sont en fait retombés en mer (Takemura 2011; Nakajima 2017). Les types de contaminants ont été l'iode-131 (dangereuse quelques semaines), le césium-134 (60 ans), le césium-137 (300 ans) (Repussard 2012). Parmi les territoires touchés, ce sont surtout les plaines rizicoles du Kantō et d'une partie du Tōhoku ainsi que la ceinture maraîchère de Tokyo qui ont été affectées.

La crise alimentaire issue de la contamination radioactive de mars 2011 s'incrit dans le processus de prise en compte de la qualité sanitaire des aliments et sa perception par les consommateurs. Les crises entendues dans ce sens sont donc " des phases d'exacerbation et de médiatisation d'une situation considérée comme dangereuse, avec la mise en scène de controverses et de débats sur la nature du danger, les modalités de l'évaluation du risque et les conditions de sa gestion préventive et corrective" (Poulain 2012: 1244). Celle provoquée par l'accident de la centrale de Fukushima, même si elle est exceptionnelle, peut s'analyser de cette manière, selon le modèle élaboré par le sociologue de l'alimentation Jean-Pierre Poulain, qui met en évidence une évolution en cinq phases (fig. 01). 


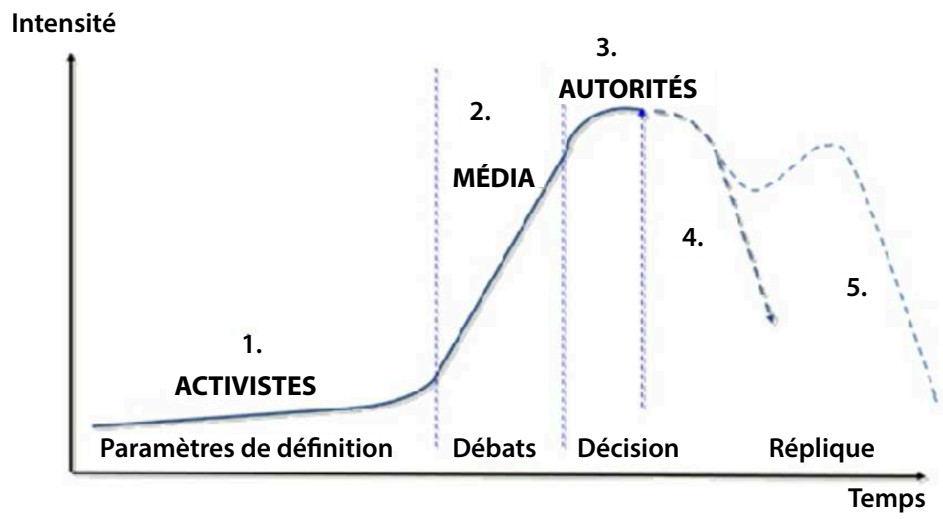

1. Phase de latence où certains éléments du risque sont connus, mais où le problème ne retient pas encore l'attention du grand public.

2. Phase de médiatisation et d'inquiétude qui correspond au moment où la crise devient centrale dans le débat public.

3. Phase de prise de décision où les autorités administratives prennent en urgence des mesures pour gérer la crise et ramener la situation à la normale.

4. Phase de reflux où les décisions sont prises, comme les contrôles, les interdictions et les retraits du marché, commencent à avoir une influence.

5. Phase de retour à une période de latence avec des répliques qui nécessitent que les décisions soient revues ou complétées.

\section{Fig. 01}

La dynamique d'une crise alimentaire.

En adoptant ce cadre d'analyse, il apparaît que la crise avait en réalité virtuellement débuté bien avant l'accident de la centrale de Fukushima, dès la prise de conscience minoritaire de la vulnérabilité de l'agriculture face à un tel aléa. Malgré des alertes comme l'accident de Tokaimura 東 海村 de 1999 et celui de Kashiwazaki-Kariwa 柏崎刈羽 de 2007, le point important à retenir est que cette pollution n'avait pas été vraiment anticipée, ce qui a contribué à aggraver les craintes des consommateurs. Avant l'accident, lors de la phase de latence, il y avait certes des lanceurs d'alerte, comme les groupes antinucléaires. Il y avait aussi des choix concients et une demande de meilleure traçabilité des aliments exprimée par une minorité, 
en particulier par le réseau de l'agriculture biologique qui posait des questions plus générales, sur l'origine, les modes de production et la qualité des aliments (Ōyama 2006; Orito 2014). Cependant, il n'existait pas de réglementation sur la vente et la consommation de produits potentiellement radioactifs. Dès lors que la contamination s'est produite, elle a réduit toutes les questions de qualité de l'alimentation à leur aspect de sécurité et la géographie des lieux de production a aussi pris une importance considérable dans le choix des consommateurs.

Pour les paysans et les pêcheurs, sur le moment, la situation a été très compliquée. L'ampleur de la contamination n'était pas connue. La réalité de la gravité de l'accident non plus. Dans les premiers mois, outre de formuler des demandes d'indemnisation rapide, les paysans de l'est du département de Fukushima rencontrés par Hiroko Amemiya se souciaient surtout de savoir s'il était possible de cultiver du riz, car la saison du repiquage était toute proche. Les légumes à feuilles, cultivés juste au moment de l'accident, avaient été interdits à la vente, il était prévu qu'il y aurait des compensations, mais personne n'avait encore été indemnisé fin avril. Les témoignages montrent des drames personnels qu'aucune indemnisation n'a pu racheter, mais aussi différentes attitudes et stratégies face à la catastrophe (Amemiya 2014). Dans les territoires limitrophes de la zone d'interdiction de $30 \mathrm{~km}$, certains sont partis, d'autres ont cessé leurs activités, d'autres enfin ont fait le choix de continuer, malgré les risques et les difficultés à vendre leur production.

Le coût des dégats pour les secteurs de l'agriculture et de la pêche a été très important. En suivant les chiffres du ministère de l'Agriculture, des Forêts et de la Pêche, la triple catastrophe aurait coûté 2430 millards de yens (52\% pour le secteur de la pêche, $39 \%$ pour celui de l'agriculture et $9 \%$ pour le secteur des forêts) ${ }^{2}$. En aval de la chaîne, près des trois-quarts des exploitants du secteur alimentaire ont été touchés, au moins pendant la première année, par la baisse des ventes ou l'augmentation des prix de gros (Boonstra 2013 : 130).

2. Heisei 23 nendo shokuryō, nōgyō, nōson hakusho 平成 23 年度食料農業農村白書 (Livre blanc sur l'alimentation, l'agriculture et le milieu rural) https://www.maff.go.jp/j/ wpaper/w_maff/h23/ (dernière consultation en juin 2021). 
Le moment où la crise est devenue centrale dans le débat public s'est situé entre mars et août 2011. Une de ses caractéristiques a été notamment l'arrivée des réseaux sociaux qui a court-circuité les médias traditionnels comme la presse écrite, la radio ou la télévision. Ce fut le temps des découvertes, des indignations et de la mise en lumière des insuffisances. Ce fut aussi le moment où les positions des différents partis ont été les plus tranchées, avec d'un côté les nombreuses manifestations antinucléaires auxquelles ont participé notamment des agriculteurs et des mères de familles, et d'un autre côté les actions de soutien à la consommation des produits des régions sinistrées.

\section{Des mesures prises en urgence}

Comme, il n'existait pas véritablement de réglementation concernant l'interdiction à la consommation de produits contaminés (à part les recommandations faites par la Commission de sécurité nucléaire en 2002 après l'accident de Tokaimura de 1999) aucune disposition n'encadrait la distribution et la vente des produits (Berends \& Kobayashi 2012; Gōdo 2014) ${ }^{3}$. À la suite de l'accident, plutôt que de prôner par précaution une interdiction totale de l'agriculture d'une zone élargie, le gouvernement japonais a fait le choix risqué de limiter au minimum les zones d'interdiction et de se concentrer sur les produits avec un système de tests, ce qui signifiait fixer des niveaux maximum de contamination acceptables et s'assurer que de la nourriture trop contaminée ne se retrouve pas sur le marché.

Le 17 mars, 6 jours après le tremblement de terre, les premières mesures en rapport avec les produits de l'alimentation courante étaient ainsi annoncées, avec une modification des articles 1 et 6 de la loi sur l'hygiène alimentaire (shokuhin eisei hō 食品衛生法, Food Sanitation Act) ${ }^{4}$, amorçant le début de la troisième phase de la crise. La gravité de la situation empêchant de véritablement prendre le temps de discuter et d'apprécier l'efficacité des mesures, les

3. D’après Gōdo (2014), l'absence de règlementation résulterait en fait de choix volontaires, faits afin de ne pas inquiéter les populations sur la possibilité d'une contamination radioactive, alors que des mesures avaient été proposées en 2002.

4. MHLW, Handling of food contaminated by radioactivity (Relating to the accident at the Fukushima Nuclear Power Plant), 2011. http://www.mhlw.go.jp/english/topics/ foodsafety/dl/food-110317.pdf (dernière consultation en juin 2021). 
phases de médiatisation de la crise se sont entrecoupées, obligeant les autorités à faire des ajustements fréquents. Ainsi, lors des premières interdictions de vente, l'ensemble des produits n'était pas encore couvert. Il n'y avait, par exemple, ni de règlementation, ni de mesures prévues pour la contamination des poissons et des fruits de mer. Ces normes ont été ajoutées le 5 avril 2011 et une série de test a immédiatement débuté afin de rassurer les consommateurs et les destinataires étrangers des exportations japonaises ${ }^{5}$.

Devant les inquiétudes de la population, l'ensemble des maxima autorisés concernant les césiums 134 et 137 a ensuite été revu à la baisse à partir d'avril 2012. Le nombre de départements où les produits sont testés a également progressivement été élargi au cours de la première année (voir la carte en fig. 02).

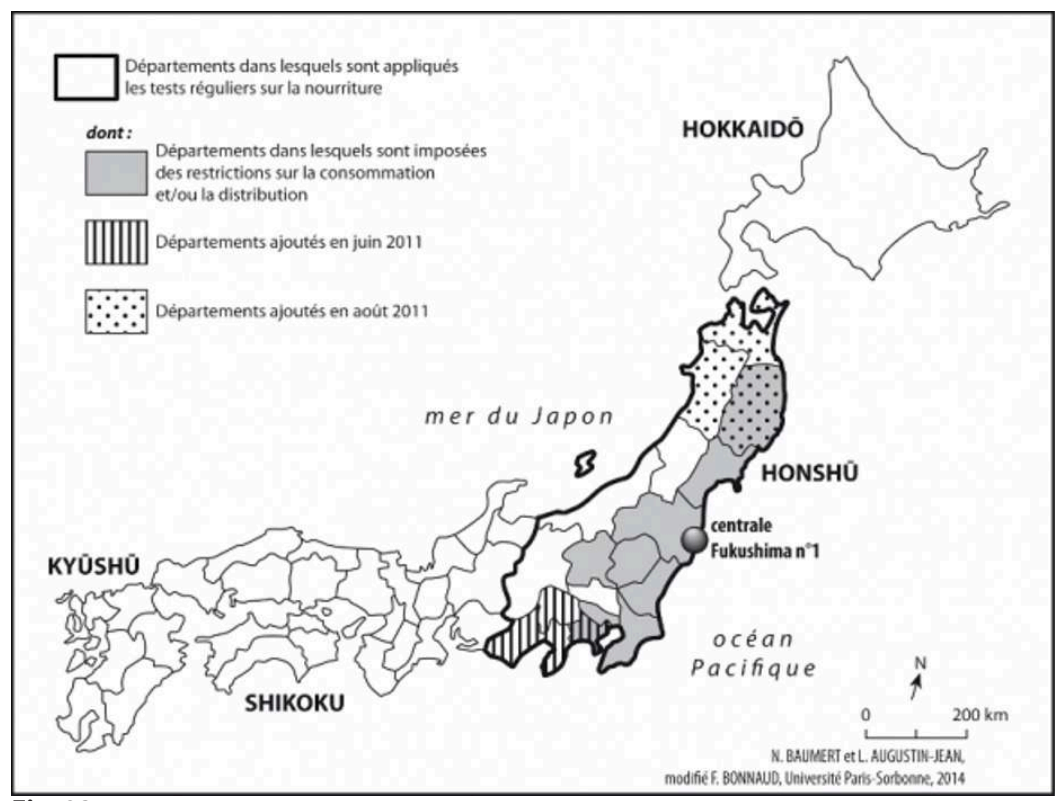

Fig. 02

Zonage des tests des productions alimentaires et des interdictions mises en place au Japon depuis avril 2011.

Source : Augustin-Jean \& Baumert (2012).

5. https://www.mofa.go.jp/files/000040303.pdf (dernière consultation en juin 2021). 
À partir de ce moment, les limites maximales autorisées par le Japon sont devenues les plus sévères au monde. Selon les produits, elles étaient inférieures de 4 à 20 fois aux normes édictées en mars 2011, largement sous les seuils admis aux États-Unis et dans l'Union européenne (par exemple pour l'eau, 10 Bq/l au Japon, 1000 dans l'Union européenne et 1200 aux États-Unis, voir le tableau 01). C'est toujours un élément important de la communication de crise, néanmoins il faut prendre en compte le fait que les règlements américains et européens sont prévus pour les situations d'urgence, afin de gérer des évacuations et non pour des populations qui restent sur place.

\section{TABLEAU 01}

Comparaison des normes de commercialisation et de consommation des denrées alimentaires concernant les valeurs de la teneur en césiums-134 et 137.

\begin{tabular}{|l|l|l|l|l|}
\hline $\begin{array}{l}\text { Valeurs autori- } \\
\text { sées en Bq/kg }\end{array}$ & Eau & Lait & $\begin{array}{l}\text { Aliments de } \\
\text { base }\end{array}$ & $\begin{array}{l}\text { Aliments pour } \\
\text { enfants }\end{array}$ \\
\hline $\begin{array}{l}\text { Japon depuis } \\
\text { avril 2012 }\end{array}$ & 10 & 50 & $\begin{array}{l}100 \text { (incluant } \\
\text { les produits } \\
\text { laitiers) }\end{array}$ & 50 \\
\hline $\begin{array}{l}\text { Japon avant } \\
\text { avril 2012 }\end{array}$ & 200 & 200 & 500 & 200 \\
\hline États-Unis & 1200 & 1200 & 1200 & 1200 \\
\hline $\begin{array}{l}\text { Union euro- } \\
\text { péenne }\end{array}$ & 1000 & 1000 & 1250 & 400 \\
\hline Codex & 1000 & 1000 & 1000 & 1000 \\
\hline
\end{tabular}

Adapté de MLHW (2012).

La mise en place des contrôles a permis de se faire une première idée de la réalité de la contamination. Le 19 mars 2011, un premier cas a ainsi été détecté dans du lait venant d'une ferme située à $30 \mathrm{~km}$ de la centrale accidentée. Par la suite, le 15 avril, sur une liste de 98 produits, 22 contenaient des taux de contamination supérieurs aux normes nouvellement édictées. Enfin, en juin, il était révélé que de la viande dépassant les taux acceptés 
avait été distribuée et parfois vendue dans 45 des 47 départements du pays ${ }^{6}$, tandis que des tests effectués au niveau international montraient une contamination de produits japonais à l'exportation dont du thé, testé en France à l'aéroport de Roissy ${ }^{7}$.

Une baisse progressive des cas détectés ainsi que des passages avérés dans la chaîne alimentaire est observable tout au long des dix ans qui ont suivi l'accident. Il y avait plus de 2000 cas en 2012, puis seulement la moitié en 2013, et moins de 500 en 2016, cinq ans après l'accident. En 2020, un seul cas concernant des champignons a été détecté (Kōsei rōdōshō 2020) ${ }^{8}$. Des problèmes sont surtout apparus pendant la première année, principalement à cause du système de contrôle qui n'était pas en place et du fait du manque d'appareils de mesure, puis il y a eu une amélioration progressive allant de pair avec la décontamination des sols, ce qui a permis une reprise des activités agricoles, même dans les communes limitrophes de la centrale accidentée.

Ces chiffres officiels sont-ils réellement rassurants? Il est impossible de savoir combien de produits sont passés (par accident, négligence ou par fraude) au travers du système de tests ni si cela a eu une conséquence sur la santé des consommateurs. Les rapports des Nations unies n'indiquent pas d'augmentation significative de maladies dues à l'alimentation (UNSCEAR 2013, 2020). Il n'y a pas non plus de signe de contamination durable due à l'alimentation des personnes dans les zones proches d'après des mesures indépendantes (Chikurin 2017), ce qui permet de penser que les mesures de restriction de commercialisation des produits dépassant les limites prévues par la règlementation ont été efficaces (Osanai et al. 2021). Des recherches sur les animaux dans la zone interdite ont néanmoins montré des taux de contamination plus élevés (Urushihara et al. 2018), ce qui indique que le risque est toujours présent et qu'une vigilance sur la consommation des aliments produits dans les zones contaminées est encore nécessaire.

6. "Colossal Blunder" on Radioactive Cattle Feed. Govt Officials Admit Responsibility for Foul-Up that let Tainted Beef Enter Nation's Food Supply ", Daily Yomiuri, 18 juillet 2011.

7. " Du thé radioactif japonais intercepté à Roissy ", Le Monde, 17 juin 2011

8. Données du ministère de la Santé, du Travail et des Affaires sociales (Kōsei rōdōshō, 厚生労働省, MHLV) disponibles sur le site https://www.mhlw.go.jp/ (dernière consultation en juin 2021). 


\section{La réaction des consommateurs : entre recherche de sécurité, soutien et oubli}

Les décisions des autorités pour gérer la crise et ramener la situation à la normale n'ont été qu'une facette de la crise alimentaire et ce sont aussi les consommateurs qui ont influencé par leurs actions l'évolution du système alimentaire. À la suite de la catastrophe nucléaire, les Japonais se sont sentis bien évidemment de plus en plus concernés par les questions de sécurité alimentaire. L'ensemble des enquêtes réalisées lors des cinq premières années le montre. Par exemple, une enquête Nikkei de début 2012 indiquait que depuis l'accident de Fukushima, $70 \%$ des personnes interrogées se sentaient alors "grandement concernées par la contamination alimentaire ${ }^{9}$. Cette prise de conscience était le reflet de l'inquiétude née de la contamination et du manque de confiance par rapport aux normes édictées et à la nature des contrôles. Non seulement les avis des experts étaient divergents, mais tout au début de la crise, les départements et municipalités avaient aussi alerté sur le fait qu'ils n'avaient pas suffisamment d'équipements pour effectuer les tests. L'attitude du gouvernement japonais lui-même a donné lieu à des critiques, puisqu'il a non seulement réagi avec retard, mais qu'il a aussi donné l'impression de vouloir cacher l'ampleur de la catastrophe.

Dans les premières années de la crise, la confusion née des différentes interprétations de la norme n'a pas augmenté le risque lui-même, mais plutôt sa perception. Cela a entraîné des comportements diamétralement opposés (soit dans le but d'aider les régions touchées, soit en étant extrêmement critiques et sceptiques envers les mesures officielles) de la part d'une partie, certes minoritaire, des consommateurs. Ces minoritées actives représentent des tendances ayant influé tant sur les ajustements faits par les autorités que par le secteur privé (Augustin-Jean \& Baumert 2014).

Une partie des consommateurs a ainsi volontairement privilégié dès le départ la consommation des produits en provenance des régions les plus touchées afin d'aider les populations et de défendre l'économie locale. Les ventes de saké du Tōhoku ont par exemple augmenté de $15 \%$ en 2011 et beaucoup de produits emblématiques ont rapidement été en rupture de

9. "Fukushima Disaster Raised Awareness of Food Safety: Polls ", Nikkei, 7 mars 2012, Nikkei Report. 
stock. Les personnes effectuant ce choix avaient des sentiments « localistes » et « loyalistes » clairement affirmés, dans le sens où leur objectif est d'aider la région touchée et le pays à se redresser. Elles n'ignoraient bien évidement pas le risque alimentaire potentiel, mais les risques sur leur propre santé leur semblaient faibles en comparaison des bénéfices qu'elles pouvaient apporter aux régions dévastées. Les campagnes de soutien se sont succédées pendant 10 ans. À la suite de la crise de la Covid-19 en 2020, les ventes en ligne sur les sites Internet ont pris le relais et génèrent toujours des revenus importants qui permettent d'aider les producteurs locaux. Ces ventes ont ainsi rapporté 2,1 milliards de yens en 2020 pour le seul département de Fukushima (Fukushimaken kōhōka 2020).

D'autres consommateurs ont aussi été très actifs, mais de façon différente des précédents. Ils ont été critiques envers l'action gouvernementale. L'engagement a été plus fort et plus précoce chez les personnes déjà concernées par la qualité agroalimentaire avant la catastrophe, comme par exemple, les membres de coopératives de consommateurs et les femmes avec des enfants en bas âge. Sur le plan individuel, elles ont mis en doute les critères existants et ont reconstruit leurs propres normes: des valeurs de césium plus basses que les normes en vigueur et/ou un espace géographique de sécurité plus élargi. Une action plus collective, générée par des associations ou coopératives déjà existantes ou par des organisations nouvellement créées, s'est ensuite superposée. Face au risque perçu, les consommateurs qui se sentaient les plus concernés se sont organisés pour protéger la sécurité alimentaire en collectant et en diffusant l'information ou en la créant. De nouvelles et anciennes ONG et coopératives ont, par exemple, acquis des équipements afin de pouvoir tester la nourriture apportée par les citoyens, dans le but de compléter et de vérifier les contrôles officiels, d'établir une cartographie des risques et de dénoncer les manquements aux règles de sécurité (Augustin-Jean \& Baumert 2014).

Le comportement des consommateurs les plus critiques illustre les conséquences de la mauvaise adéquation entre les aspirations des individus en matière de sûreté alimentaire et les prises de décisions politiques, plutôt orientées par le travail des experts. Il est d'ailleurs apparu dans cette crise comme pour d'autres, que la communication et l'information apportés par les autorités ne modifient qu'à la marge les comportements des consommateurs. Des enquêtes sur la perception du risque par des mères de familles avant et après une formation avec des experts sur les dangers 
de la radioactivité montrent en effet que la connaissance des risques n'est pas le seul critère de choix et ne diminue pas significativement les craintes (Niiyama 2019). Le rapport à l'alimentation reste en effet un invariant anthropologique désormais bien connu qui veut qu'en incorporant un aliment, le mangeur incorpore aussi sa dimension symbolique (Fischler 2001) et la symbolique du nucléaire est dans ce cas trop forte pour véritablement pouvoir apaiser les craintes des consommateurs. Une part significative des consommateurs (autour de $20 \%$ ) indiquaient ainsi lors des enquêtes effectuées au début de la crise qu'ils refusaient d'acheter des produits issus des départements les plus touchés et cela même si des contrôles négatifs leurs étaient présentés (JA kyōsai sōgōkenkyūjo 2012).

Ces conséquences ont abouti à la reconstruction de systèmes de sécurité alimentaire qui ont également été récupérés et institutionnalisés par le secteur privé. En novemmbre 2011, le groupe Aeon/Jusco a été le premier à mettre en place une première norme privée et des tests destinés à garantir que les produits vendus dans ses magasins auraient "zéro cesium ", avant d'établir finalement une limite inférieure à $30 \mathrm{~Bq} / \mathrm{kg}^{10}$. De même, alors que dans un premier temps, les supermarchés de Tokyo avaient fait la promotion des produits de Fukushima, Miyagi et Iwate, ils ont ensuite changé de stratégie et se sont au contraire adaptés à la demande des consommateurs les plus inquiets (Kimura 2012). De nombreuses municipalités ont également suivi cette nouvelle norme, en particulier pour leurs cantines scolaires ${ }^{11}$.

Dans la quatrième phase de la crise, celle du reflux, les actions de ces groupes sont devenues de moins en moins visibles et médiatisées, même si elles ont eu un rôle important dans la création des normes et dans la perception de la catastrophe. Malgré les inquiétudes souvent exprimées, la majorité de la population n'a en réalité pas vraiment modifié son mode de consommation sur la durée. Cela ne signifie pas pour autant que les personnes ne se souciaient pas de leur sécurité alimentaire; elles ont suivi les décisions des autorités et leur attitude a davantage consisté à ne pas trop

10. https://www.aeon.info/news/2011_2/pdf/111108R_1.pdf (dernière consultation en juin 2021).

11. "Localities to Ramp up Radiation Testing of Food ", Nikkei, 29 mars 2012, Nikkei Report. 
chercher à savoir, de manière à réduire l'anxiété provoquée par leur inaptitude à évaluer correctement le risque.

La vigilance face à la consommation de produits contaminés baisse d'année en année. Pour le département dont le nom est potentiellement le plus anxiogène, Fukushima, les enquêtes de consommation montrent que les personnes refusant d'acheter des produits du département sont en baisse régulière. De 19,6 \% en 2013, le pourcentage est ainsi passé à 12,5\% en 2019. Un fait plus étonnant est que la connaissance des personnes interrogées sur la réalité et la nature des tests diminue aussi. Dans l'Ouest du Japon, hors de la zone où sont effectués les tests sur la nourriture, seuls $44,8 \%$ des personnes interrogées sont encore avertis de leur existence (Fukushimaken kōhōka 2020).

\section{Une interrogation subséquente à la crise : le rapport à la Terre via la médiation alimentaire a-t-il changé?}

Au printemps 2011, il était difficile de voir la ligne directrice des autorités japonaises sur les questions d'agriculture et d'alimentation, mais avec le recul, et malgré les tâtonnements et les doutes qu'il y a forcément eus, elle apparaît à présent claire : il s'agissait de surmonter la crise et revenir le plus rapidement possible à la normale. Le but était donc de rassurer le plus possible les consommateurs et d'éviter trop de drames chez les producteurs. Aucun de ces deux objectifs n'a réellement été atteint, mais la crise, malgré son aspect historique, reste finalement une crise alimentaire dont les différentes phases peuvent s'identifier selon le modèle présenté en fig. 01 et qui, en 2021, se situe dans celle du retour à la normale.

Dix ans après l'accident, l'agriculture dans les zones les plus contaminées proches de la centrale accidentée reste encore interdite, mais ces espaces ne représentent qu'une très faible partie des terres agricoles. Dans l'ensemble des départements, les volumes de production ont peu changé par rapport à avant 2011. Visuellement, hors des zones interdites ou à accès limité, il y a finalement peu de friches et peu de terres laissées à l'abandon.

À Fukushima, dans les municipalités de Minamisōma 南相馬 ou Iitatemura 飯舘村, des parcelles expérimentales ont été mises en place pour reconquérir les terres petit à petit en permettant d'abord aux agriculteurs de revenir cultiver sans y habiter (Ishii \& Morlan 2014), puis de 
reprendre leurs activités avec les premières levées d'interdiction en 2016. À d'autres endroits, comme dans la plaine de Sendai, le développement d'usines d'agriculture hors-sol a permis de reprendre des activités agricoles sans avoir recours à des terres rendues impropres aux cultures dans les zones où le tsunami avait rendu excessive la teneur des sols en sel.

La remise en culture des terres a aussi été l'occasion de profiter de dérégulation de la loi agraire de 2009 pour faire avancer des projets financés par des entreprises multinationales qui se sont finalement confrontées violement au modèle agricole traditionnel et familial comme le montre le cas de la ferme Michisaki みちさき dans le département de Miyagi. Cette ferme, située près de la côte, à l'Est de Sendai, était censée faire collaborer des producteurs locaux et des entreprises comme IBM, Mitsubishi et Kagome avec l'apport de subventions publiques pour la production de légumes sous serres, mais les investissements ont en fait créé des mécontentements chez les acteurs locaux, puisque le projet d'implantation des serres concurrençait la production de riz que certains producteurs sinistrés souhaitaient redévelopper (Sekine 2020). D’une certaine manière, la recontruction du Tōhoku a été vue par les autorités comme une opportunité pouvant servir d'exemple pour encourager une évolution libérale du modèle agricole japonais à l'échelle nationale (Boonstra 2013), mais la tentative n'a pas été concluante et la tendence générale montre finalement que l'essentiel des efforts a été déployé pour revenir à la situation antérieure.

Pour les paysans, ceux qui ont choisi de rester et de continuer leurs activités, malgré la fin de la plupart des aides en 2018 sont un peu contraints de jouer leur rôle de "décontaminateurs " des sols et leur action, qui permet non seulement de maintenir les terres agricoles mais également de préserver les paysages, est finalement peu reconnue. Le système de compensation financière a également été source d'iniquité et les agriculteurs des régions situées juste à l'extérieur des zones d'évacuation ont reçu peu d'aides, bien que leurs activités aient également été affectées par des dépôts radioactifs et qu'ils soient toujours confrontés à des difficultés similaires ${ }^{12}$. Une différence de prix de 10 à $20 \%$ plus bas que la moyenne des prix du marché est toujours observée en 2019, notamment pour le riz, les productions fruitières

12. "Tepco's Compensation for 3/11 Victims Has Made Matters Worse for Many ", The Japan Times, 13 avril 2018. 
de pêches ou le bœuf de Fukushima (MAFF 2019). Certains secteurs ont également adopté des stratégies de contournement, dont le but est d'annuler la réputation négative conférée à leurs produits. Par exemple, la viande de "bœuf de Fukushima " est vendue comme kokusan gyū 国産牛 (viande de bœuf japonaise), ce qui permet de maintenir les niveaux de vente, mais à un prix inférieur (Maître et al. 2020).

Le volume des exportations du département de Fukushima en chiffres montre néanmoins un rattrapage impressionnant durant les cinq dernières années pour les productions emblématiques comme le riz et les pêches. Après une chute brutale en 2011 et une reprise lente jusqu'en 2016, les volumes sont, dix ans plus tard, plus importants qu'avant l'accident (Fukushimaken kōhōka 2020). Le département est redevenu le 4e producteur de riz du Japon en volume (MAFF 2020), position qu'il occupait avant l'accident de la centrale. Le sentiment qu'on retient est donc celui du retour à la normale qui est à la base de la communication officielle : le Japon a surmonté l'épreuve.

Un tel constat dépend bien entendu de l'échelle d'analyse et de l'espace étudié. Pour les agriculteurs et les pêcheurs des communes les plus touchées, la situation n'est clairement pas redevenue normale : de la pollution subsiste, même si la radioactivité est, sur les terres agricoles, inférieure aux normes. Plus généralement, la question qu'il faut poser à l'échelle nationale est de savoir si la situation d'avant était vraiment normale. Est-ce que l'agriculture japonaise n'était déjà pas à repenser? Est-ce que la nature, la qualité et la traçabilité de l'alimentation étaient si parfaites qu'il faille simplement revenir à la situation d'avant 2011 en ajoutant simplement des tests de contamination pour se satisfaire de ce constat?

En fait, malgré les réponses techniques apportées, la vulnérabilité des systèmes de production et d'approvisionnement alimentaires du Japon, finalement toujours aussi complexes et le plus souvent coupés du lien avec leur environnement immédiat, n’a pas été repensée. La contamination nucléaire issue de l'accident de la centrale de Fukushima se situe donc dans la lignée des crises comme celles de la vache folle ou des grippes aviaires. Certes, elle constitue un nouvel échec de la modernité, elle a permis de replacer l'horizon de la catastrophe dans le champ des possibles et a renforcé les convictions des groupes minoritaires qui, depuis longtemps, prônaient un retour au local, l'abandon de l'agriculture dite productiviste et la sortie d'un système basé sur la croissance infinie (Sabban 2006; Baumert 
\& Guichard-Anguis 2014; Lagane 2016), mais il n'y a pas eu de rupture. Seuls quelques ajustements ont été effectués et le rapport de la société à son alimentation et à son agriculture n'a pas été repensé, ce qui permet au spécialiste d'agriculture Gōdo Yoshihisa 神門善久 de qualifier la situation de l'agriculture japonaise comme déprimante au regard de ce problème (Gōdo 2012).

Un aperçu des conventions portant sur la qualité des aliments permet de mieux comprendre la nature du problème. Quatre niveaux de confiance sont généralement adoptés : le "bio nature ", les indications d'origine, la personnification de la relation avec les producteurs, le label technicoscientifique (Poulain 2012). Ils couvrent tous les aspects de la notion de qualité, de la qualité sanitaire aux normes et à la qualité sociétales. Seule une combinaison des diverses approches permet d'instaurer une solide relation de confiance entre les producteurs et les consommateurs, de sorte que l'on peut parler de paradoxe de la contamination radioactive à ce sujet. En effet, pour répondre à la contamination radioactive, c'est le dernier critère, le label technico-scientifique, qui a été mis en avant par les autorités (tandis que beaucoup de consommateurs ont aussi utilisé l'indication de provenance qui a eu un aspect rassurant et qui l'a complété). Toutefois, en prenant du recul, il faut bien se souvenir que c'est cet aspect technicoscientifique non maîtrisé sur le plan du nucléaire qui est à l'origine de la crise. On peut donc voir la résolution de la crise comme une obligation de confiance dans un mode de fonctionnement technico-scientifique qui a justement dysfonctionné. La question qui se pose est donc celle du rapport de la société à son environnement, à la Terre, dont la médiation alimentaire est une partie essentielle et quotidienne à la source de la vie humaine (Orito 2014) et, en cela, se satisfaire d'un retour à la normalité d'avant la crise peut être qualifié d'anormal. 


\section{Bibliographie}

\section{Amemira Hiroko 2014}

« Notes de terrain : Portraits de quelques paysans et acteurs du système teikei après la catastrophe de Fukushima », Géographie et cultures, 86 : 119-132.

Augustin-Jean Louis \& Baumert Nicolas 2014

«Les réactions des consommateurs japonais suite à la contamination nucléaire de mars 2011 et leurs conséquences sur le rapport au territoire », Géographie et cultures, 86 : 49-64.

Apfelbaum Mariam (dir.) 1998

Risques et Peurs alimentaires, Paris, Odile Jacob.

Baumert Nicolas et Guichard Anguis Sylvie 2014

"Désastre et alimentation, le défi japonais », Géographie et cultures, 86 : 5-12.

Berends Gijs \& Kobayashı Megumi 2012 «Food After Fukushima, Japan's Regulatory Response to the Radioactive Contamination of its Food Chain », Food and Drug Law Journal, 67 (1) : 51-64.

\section{Boonstra Carla 2013}

«Rebuilding farming in Tohoku: A new frontier for Japanese agriculture? », in Al-BADRI Dominic \& Berends Gils (dir.) After the Great East Japan Earthquake: Political and Policy Change in Post-Fukushima Japan, Copenhage, NIAS Press : 129-148.

\section{Chikurin NPO 2017}

Hōjin shimin hōshanō kanshi sentā 法人 市民放射能監視センター (Centre citoyen de surveillance de la radioactivité), database.

http://chikurin.org/?page_id=10 (dernière consultation en mars 2021).

FerRIĖRE Madeleine 2002

Histoire des peurs alimentaires, Paris, Éditions du Seuil.

Fischler Claude 2001

L'Homnivore, Paris, Odile Jacob.

GōDo Yoshihisa 神門善久 2012

Nihon nōgyō e no tadashii zetsubō hō日 本農業への正しい絶望法 (De la bonne manière de désespérer de l'agriculture japonaise), Tokyo, Shinchōsha 新潮社.

\section{Gōdo Yoshihisa 2014}

«Regulation Values for Radioactive Materials in Food After the Fukushima Nuclear Power Plant Accident », Agricultural Policy Platform (FFTC-AP). https://ap.fftc.org.tw/article/732 (dernière consultation en juin 2021).

IsHII Keiichi \& MorLans Shantala 2014 « La reprise des activités agricoles dans les régions contaminées après l'accident de Fukushima », Géographie et cultures, $86: 65-82$.

\section{JA kyōsai sōgōkenkyūjo JA共済総合 研究所 (Institut de recherche de la mutuelle d'assurance JA) 2012 Kyōsai sōken repōto 2012.10 共済総研L ポート 2012.10 (Rapport d'octobre 2012). https://www.jkri.or.jp/PDF/2012/}


Rep123ueda.pdf (dernière consultation en mars 2021).

\section{Kımura Aya 2012}

«Standards as Hybrid Forum:

Comparison of the Post-Fukushima Radiation Standards by a Consumer Cooperative, the Private Sector, and the Japanese Government », International Journal of Sociology of Agriculture and Food, 20 (1) : 11-29.

\section{Kōsei rōdōshō 厚生労働省 (Ministère de la Santé, du Travail et des Affaires sociales)}

Rapports annuels.

https://www.mhlw.go.jp/ (dernière consultation en juin 2021).

\section{LAGANE Jean 2016}

Minamata et Fukushima, de la nature des catastrophes, Marseille, Editions Gaussen.

Maître Mélanie et al. 2020

«The Management of Contaminated Goods in Japan Since the Fukushima Accident », Radioprotection, 55.

https://www.radioprotection.org/articles/ radiopro/abs/2020/01/radiopro200001/ radiopro200001.html (dernière consultation en juin 2021).

Nakajima Teruyuki et al. 2017 « Model depiction of the atmospheric flows of radioactive cesium emitted from the Fukushima Daiichi Nuclear Power Station accident», Progress in Earth and Planetary Science, 4 (2). https://doi.org/10.1186/s40645-0170117-x (dernière consultation en juin 2021).

\section{Nirama Yoko 2019}

«Essential Elements for Interactive Risk Communication in the Food-Related Emergencies: A Model and Experiments on the Health Effects of Radioactive Substances ", in Poulain Jean-Pierre et Augustin-Jean Louis (dir.), Risk and Food Safety in China and Japan: Theoretical Perspectives and Empirical Insights, London, Routledge : 101-124.

Nōrin suisanshō 農林水産省 (Ministère de l'Agriculture, des Forêts et de la Pêche, MAFF)

Rapports annuels.

https://www.maff.go.jp/index.html

(dernière consultation en juin 2021).

Nōrin suisanshō 農林水産省 (Ministère de l'Agriculture, des Forêts et de la Pêche, MAFF) 2019

Heisei 30 nendo Fukushima kensan nōsanbutsutō ryūtsū jittai chōsa hōkokusho gaiyō 平成30年度福島県産農 産物等流通実態調査報告書概要 (Résumé du rapport de l'enquête 2018 sur la distribution des produits agricoles du département de Fukushima).

https://www.maff.go.jp/j/shokusan/ryutu/ attach/pdf/190329-10.pdf (dernière consultation en juin 2021).

\section{ORITO Etona 2014}

« Les teikei - les précurseurs au Japon de l'agriculture biologique - face à la catastrophe nucléaire de mars 2011 ", Géographie et cultures, 86 : 83-99.

Osanal Minoru et al. 2021

« Estimation of Effect of Radiation Dose Reduction for Internal Exposure by Food 
Regulations under the Current Criteria for Radionuclides in Foodstuff in Japan Using Monitoring Results », Foods, 10, 691.

https://doi.org/10.3390/foods10040691 (dernière consultation en juin 2021).

Poulain Jean-Pierre 2012

"Risques et crises alimentaires », in PoulaIN Jean-Pierre (dir.), Dictionnaire des cultures alimentaires, Paris, PUF : 1243-1261.

\section{Ōyama Toshio 2006}

"Le consommateur japonais face aux produits biologiques : leçons d'un système de commercialisation diversifié », Ebisu. Études japonaises, 35 : 9-29.

\section{TAKEMURA Toshihiko et al. 2011}

«A Numerical Simulation of Global Transport of Atmospheric Particles Emitted From the Fukushima Daiichi Nuclear Power Plant », SOLA, 7 : 101-104.

https://doi.org/10.2151/sola.2011-026 (dernière consultation en juin 2021).

\section{UNSCEAR 2020}

Levels and Effects of Radiation Exposure Due to the Accident at the Fukushima Daiichi Nuclear Power Station: Implications of Information Published Since the UNSCEAR 2013 Report, United Nations Scientific Committee on the Effects of Atomic Radiation.

https://www.unscear.org/docs/ publications/2020/UNSCEAR_2020_ AnnexB_AdvanceCopy.pdf (dernière consultation en mars 2021).
URUSHIHARA Yusuke et al. 2018

"Haematological Analysis of Japanese Macaques (Macaca Fuscata) in the Area Affected by the Fukushima Daiichi Nuclear Power Plant Accident », Scientific Report, 8, 16748.

https://doi.org/10.1038/s41598-01835104-0 (dernière consultation en juin 2021).

\section{Fukushimaken kōhōka 福島県広報課} (Division des relations publiques du département de Fukushima) 2020 Fūhyō/fūka taisaku no genjō to kadai 風評·風化対策の現状と課題 (Mesures à prendre pour éviter l'oubli, les erreurs et contresens).

https://www.pref.fukushima.lg.jp/ uploaded/attachment/340912.pdf (dernière consultation en mars 2021).

Repussard Jacques (dir.) 2012 Fukushima, un an après: premières analyses de l'accident et de ses conséquences, rapport IRSN/ DG/2012-001.

\section{SabBan Françoise 2006}

«Histoire et sociologie de l'alimentation ", Ebisu. Études japonaises, 35:3-8.

\section{Sekine Kae 2020}

« Néolibéralisation de la politique agricole au Japon et contradictions entre modèles agricoles », in GASSELIN Pierre et al., Coexistence et confrontation des modèles agricoles et alimentaires : un nouveau paradigme du développement territorial?, Versailles, Éditions Quae : 229-340. 
